\title{
Some control-related issues in mini-robotics for endoluminal surgery
}

\author{
Philippe Poignet and Ahmed Chemori and Nabil Zemiti and Chao Liu
}

\begin{abstract}
This paper introduces some issues related to the development of robotics for endoluminal surgery from control point of view. Endoluminal surgery are incisionless procedures performed through natural orifices within the natural pathways. New devices are then required to achieve these new surgical procedures. Besides the development of new devices, control issues arise in both technological and theoretical aspects. The paper presents some of them and we propose a teleoperation architecture that has already been tested for needle insertion that could be used for teleoperated endoluminal surgery especially for instance for biopsies or anastomoses.
\end{abstract}

\section{INTRODUCTION}

Endoluminal Surgery or Natural Orifice Translumenal Endoscopic Surgery (NOTES) are incisionless procedures performed within the natural pathways (mouth or other natural orifices) or by creating an opening in the lumen to access to abdmoninal organs. Operating through the bodys natural orifices should offer faster recovery times, less scarring and less pain, which could lead to reduced hospitalization.

Then since recently, endoluminal interventions are evolving quickly and different major procedures have emerged through endoscopic treatment. The gastroesophageal reflux disease (GERD) represents the earliest application of this new surgical procedure [25]. But various applications have been reported during the last decade including endoscopic mucosal resection [19] with a mucosal ablation depth of 600 microns to 700 microns [28], endoluminal gastroplication or radiofrequency energy delivery to the gastroesophageal junction. Works in [26] have also been reported showing the promise of endoscopic therapy for pancreatic necrosis and pancreatic abscess. Hiatal hernia repair was also performed in a pig model [4] using endoscopic ultrasound (EUS) guidance.

The feasibility of endoluminal therapies has also been demonstrated for the transgastric approach to the peritoneal cavity for diagnostic and therapeutic purposes. In [8], [20] for instance, the authors report several experiments such as liver biopsies, manipulation of intraabdominal organs, ligation of fallopian tubes, and transgastric cholecystectomy that have been performed in pigs.

The research is now focused on innovative designs and engineering improvements that have the potential to facilitate these interventions. In this context, robotics plays a key role and the objective of the paper is to introduce the issues from the control point of view which are both theoretical

This work is supported by FP7 ARAKNES ICT-2007.3.6 European project

P. Poignet, A. Chemori, N. Zemiti and C. Liu are with LIRMM, UMR 5506, Univ. Montpellier 2 - CNRS, 161 rue Ada, 34392 Montpellier, France poignet, chemori, zemiti, liuelirmm. fr and technological, and have appeared during development of this new technology.

The Natural Orifice Surgery Consortium for Advancement and Research (NOSCAR, [www.noscar.org]) identifies several wish list of capabilities or requirements depending on the application. For instance in case of NOTES anastomoses, this list highlights different aspects such as length, flexibility, navigation, vizualization, remote control, feedback, permit suturing (large channel endoscopes), ergonomics / human factors (one person operation).

The paper is then organized as follows. In the next section, we will introduce existing devices that are already commercialized or prototypes developed in robotics labs. Section III presents some difficulties associated to actuation, sensing, transmission as well as fixation problems. Section IV describes the challenges from the control point of view as well as the teleoperation architecture that has been developed for minimally invasive surgery and tested in case of needle insertion such as for biopsy or suturing cases . Experimental results highlight the efficiency of the approach that could then be used for tethered robotized endoluminal surgery. The paper ends with conclusion.

\section{A BRIEF REVIEW OF INSTRUMENTS DEDICATED TO ENDOLUMINAL SURGERY}

During the last ten years, many systems have been patented such as the "method and apparatus for minimallyinvasive fundoplication" proposed by M. El Gazayerli (Patent US6159146) in 2000 and several devices have already been developed to fulfill the requirements of these new surgical procedures.

In the following, we present a brief review of existing systems that have been classified into two categories depending on the actuation or the level of autonomy. In the first set, the user will find steerable instruments and in the second more sophisticated devices actually developed in the laboratories based on the principle of autonomous robots.

\section{A. Towards a classification of sophisticated NOTES instru- ments}

1) Steerable instruments: As identified by NOSCAR, a key requirement of NOTES surgery is to provide the surgeon with a stable surgical platform that will support and guide the flexible endoscope and instruments. Indeed, in the abdominal cavity, endoscopes alone are usually free-floating and hence unstable, limiting the physician's ability to perform surgery. For this reason, USGI Medical [www . usgimedical . com] develops the Medicals ShapeLock Endoscopic Guide and 
ShapeLock Cobra, a multilumen ShapeLock Guide for facilitating endoluminal and transgastric surgery [21]. The Cobra device offers the benefit of a stable platform for triangulation of instruments and camera and the ability to use multiple instruments at the same time. Olympus Medical Systems Corp. [www.], another competitor in this area, proposes a multibending therapeutic endoscope called R-scope used for transgastric cholecystectomy [10]. In their experiment, the R-scope, equipped with 2 actuated instrument channels (one allows vertical elevation, the other allows a horizontal swing movement) was used to position a knife for the most ideal dissection. The Endocinch suturing system [www . endocinch.com] is another example of a simple device that will help the doctor to place a series of stitches in the lower esophagus to create a pleat in the sphincter.

Although all these studies and products demonstrate the feasibility of the endoluminal approach, they also emphasize limitations of the present techniques, for instance difficulty in exerting sufficient forward force. Most of them are also cable-driven and guided or controlled by turning a wheel limiting the mobility capability or the dexterity of the device inside the cavity. Therefore the need of more autonomous robot becomes clearly identified.

2) Autonomous robot: To overcome the aforementioned limitations, for instance to offer a stable platform for vizualization and to increase the dexterity of the instrument, the authors in [16] present a miniature in vivo robot that consists of two prismatic arms connected to a central body by a rotational shoulder joint. One arm has a forceps and the other hold a cautery end-effector. The central bar is equipped with stereoscopic vision. Thanks to its shape, the robot may be inserted into the peritoneal cavity through the upper gastrointestinal tract approach.

\section{B. The ARAKNES concept}

The ARAKNES (Array of Robots Augmenting the KiNematics of Endoluminal Surgery) platform, developed in the framework of a european project [www.araknes.org], extends the concept introduced in [16] by giving the surgeon the possibility to manipulate a gastric robotic platform constituted of an array of cooperative robots including assistive and operative robots that will be ingurgitated through the esophagus as illustrated in figure 1. Assistive robots may be viewed for instance as grasping systems or robot equipped with vision and operative robots may be active instruments with suturing or cutting devices. The robots are then bimanually telemanipulated by the surgeon through a remote haptic interface. This concept fulfills then at least two of the NOSCAR requirements: possibility to include remote control of the surgical instrument and feedback through contact interaction information coming from inside the cavity.

In this context, several technological as well as theoretical issues arise, as introduced in the following sections.

\section{TECHNOLOGICAL ASPECTS ON CONTROL IN ENDOLUMINAL SURGERY}

The specifications of the new robotized instrument (architecture, size, Degrees of Freedom (DoFs), workspace,

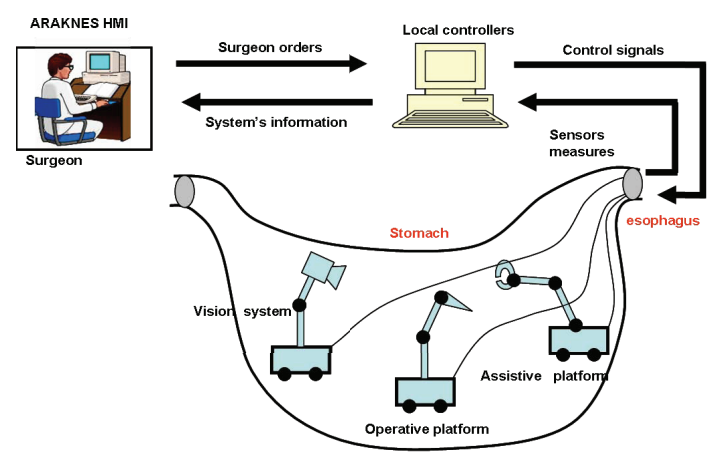

Fig. 1. Assistive and operative platforms in the ARAKNES architecture

...) for endoluminal surgery have to be considered mainly according to the pathways used for introducing instruments and to the aimed surgical task. Indeed, access to the organ is limited by the size and the complex geometry of the insertion pathway. This makes it difficult to insert multiple instruments simultaneously through one orifice.

If the aimed task is, for example, a biopsy on the stomach wall, the customized robot has to be inserted trans-orally to reach the stomach. This imposes a limit on the size and the flexibility of the robot to allow the latter to freely rotate for insertion through the complex geometry of the esophagus. Here, since the esophagus is approximately $15 \mathrm{~mm}$ diameter large for normal subjects [31], to avoid injuries, the overall robotized instrument should be of less than $15 \mathrm{~mm}$ diameter.

Besides these size constraints, one has to overcome first challenging technological problems concerning the robot architecture and its components such as actuators and sensors. Power and data transmission considerations, biocompatibility, sterilization, etc, have also to be taken into account.

\section{A. Actuators and sensors}

1) Actuators: Different solutions can be proposed such as 'on-board actuation', 'remote actuation', or combined 'on-board/remote actuation'. For on-board actuation, i.e. the actuators are placed inside the patient body, problems of miniaturization and amount of the developed force at contact have to be considered. Indeed, the developed interaction force necessary for a puncture/suturing gesture, for example, has to be greater than $10 N$ [36].

In the literature, several existing technologies might answer these problems : Shape Memory Alloys (SMA) actuators [17], Electro-Active Polymers (EAP) actuators (www.micromuscle.com), [9], [27], piezoelectric motors and electromagnetic motors [33]. However, considering the size imposed by the endoluminal surgery, the most mature and efficient technology to date (in term of the amount of developed interaction force) is piezoelectric and electromagnetic. Examples of such commercially available solutions are the micromotors from Faulhaber group (www.faulhaber-group.com) or from Namiki Precision www. namiki.net and the piezo micromotors from 
New Scale Technologies, Inc. (www.newscaletech. com).

In addition, if a large number of DoFs is chosen, problems of flexibility and backlashes due to the weight of the overall system should be considered.

Remote actuation offers the possibility of placing the actuators outside the patient body implying then much less miniaturization, power and data transmission constraints. In this case, the power transmission can be done pneumatically, hydraulically (using sterile water for example) or through cables. The main feature of this solution is that it offers a large developed force at the robot end-effector while having no limit on the size of the chosen actuators.

One can however combine both on-board and remote actuation solutions. The on-board actuation can be used to move the DoFs that don't need large interaction forces (rotate a camera for example). The remote actuation for the rest (suturing, puncturing, etc).

2) Sensors: One of the requirements for the robot is to assist the surgeon in his/her gestures providing him/her with sensing and display functions, improving thus the surgeon's ability to sense and control the interaction forces.

One of the ARAKNES project objectives is to develop a force feedback teleoperated system. Visual feedback, position and force informations are thus mandatory to be used as inputs in the robot controller.

Miniaturized position sensors already exist (potentiometers, optical encoders or hall-effect sensors) and should not be a big challenge to deal with. This information will be used in the robot position control loop. This allows the robot to perform autonomous or teleoperated tasks.

To offer a visual feedback to the surgeon, miniaturized passive cameras that can be inserted inside the patient body are now available in the context of endoluminal surgery. One can cite for example the PillCam ${ }^{T M}$ from Given Imaging or the Endo-Capsule from Olympus.

Note that the visual feedback can also be exploited as a position measurement to implement a visual servoing control scheme for the robot. This enables to control the robot with more advanced control strategies such as point to point autonomous position control.

To measure and control the interaction contact forces, one needs to use a miniaturized force sensors. Different technological solutions could then be used such as strain gauges, piezo-resistive, optical sensor [22] or electro-active polymer based sensors [27]. However, the MEMS micro force sensors like the ones presented in [32], [29], [13], have to be considered with interest to be implemented and adapted for the endoluminal surgery context.

\section{B. Fixation challenges}

One of the most important factors considered in the design of a robotic platform is providing a stable robot adhesion at the stomach wall in order to perform the required forces.

Different solutions presented in the literature can be considered. For instance, external magnetic attachment and positioning using magnetic handle, or needles and hooks configuration to attach the robot physically to the inner abdominal wall were presented in [15]. Dario et al. have recently described an endoscopic pill with an active locomotion system that uses "legs" to push against the gastrointestinal walls [2], [3], and a clamping system that uses shape memory alloys [18].

\section{Wire or wireless}

In the context of robotized endoluminal surgery, data and energy transmission is an important issue to consider. If the on board solution is chosen, wire (tethered robot) or wireless solution could be chosen depending on the available technology.

The tethered solution seems to be the simplest one if the dimension of the data/energy cables are compatible with the dimension of the patient natural orifice.

In wireless solution, one has to consider this solution since wires may constitute a channel for infection and encumber the insertion pathway.

Wireless data transmission is less complicated than wireless power transmission. For intra-body data exchange, several solutions can be considered such as WiFi, Bluetooth or RFID [34], [1].

Wireless intra-body power transmission has more challenging issues : coil design and placement, low efficiency, power dissipation in surrounding tissue, etc. However, up to date there exist few methods for wireless energy propagation that might be considered to be adapted for endoluminal applications: magnetic induction, magnetic resonance coupling, radio transmission, etc [11]. These solutions can be combined with on board rechargeable batteries (integrated thin film LiPON batteries, for example) to be recharged wirelessly. Here again, all these technologies are of interest but deep adaptations to endoluminal robotic surgery are needed.

\section{THEORETICAL AND PRACTICAL ASPECTS ON CONTROL IN ENDOLUMINAL SURGERY}

Controlling the array of robots that will be used for the ARAKNES platform may require different control modes from autonomous to teleoperated control modes with switching between control modes or between different robots.

So far, few works can be found in the litterature on autonomous control issues for NOTES robots except maybe for autonomous locomotion control such as the works presented in [23], [24].

Regarding the switching issue, two different aspects may be envisaged either from a technical point of view or from the control point of view. For the technical part, it may be solved with the classical use of button or pedal that requires from the surgeon to have a free hand or foot or it may also be solved less classically with eye tracking as proposed at Imperial College of London [30]. Besides this technical point, the stability problem in terms of control also arises when dealing with swtiching. Extensive litterature may be found in the community working on hybrid system [6]. 
The starting point of our development is the synthesis of a good teleoperated control architecture i) that will offer the surgeon a remote control of his/her instruments, ii) that will give him/her feedback information from what happens inside, especially from the interaction and finally iii) will allow him to perform anastomoses or biopsies for instance. In the following, after giving some details about teleoperation architecture, a promising candidate for a teleoperation scheme is introduced. This scheme has been already tested for needle insertion and could be used for endoluminal surgery.

\section{A. Teleoperated control mode}

The first works on teleoperation have been focused on static capabilities and kinematics. The inertia and damping have been considered but from an energy point of view, where the objective was to minimize the effort needed to accomplish the teleoperation task. Initially, two basic teleoperation architectures have been proposed [14], [7], namely:

- position-position teleoperation architecture, and

- position-force teleoperation architecture.

In position-position teleoperation architecture, the master position is transmitted as a command to the slave servo controller, and slave position is returned to the master as a position command. This makes sense if the position controllers have good tracking capabilities, since the master and slave will closely follow each other. However, master and slave robots are interconnected in a feedback loop, and the dynamics of the closed loop system must also be considered. It is worth to note that for this architecture, an accurate position control system on the master makes the system feel sluggish in free space motion, since the lags between master and slave position movements cause large reaction forces to be supplied to the operator.

In the position-force architecture, the idea is also to transmit master's positions as commands for the slave. However, the interaction force at the slave is sent back directly as a reaction force to the master. If the slave reproduces with faithfulness the master motions, and the master feels accurately the slave forces, the operator should experience the same interaction with the teleoperated task as would the slave. But this architecture does not address the dynamics of the interconnected system. Moreover, in this force reflection architecture, stability is often a problem unless the force transmitted to the master is significantly attenuated.

Later on, other works have been proposed dealing with bilateral teleoperation, focusing more on dynamics and stability and leading to a variety of proposed architectures. For instance, robust controller based on small gain theory has been proposed for force-force teleoperation architecture [12].

However the issue of transparency, which provides the human operator with a feeling of the remote environment and which is one of the key properties in surgical robotics that a surgeon may expect from the robot, has not been addressed in the above proposed teleoperation architectures. For that reason, other works have concentrated on performanceobjectives for architecture design based on for instance specifying network theory hybrid parameters [5]. However,

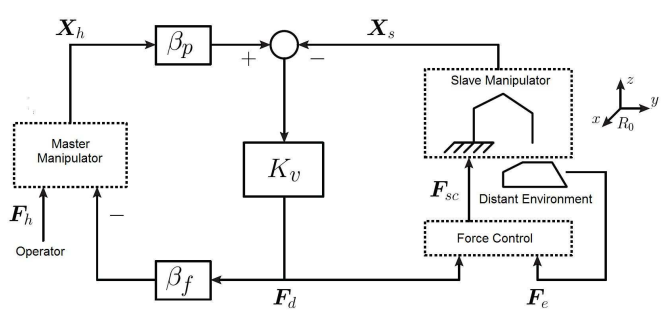

Fig. 2. Position-position teleoperation architecture.

the obtained architecture requires infinite gains to ensure accurate transparency, which is obviously unacceptable. Other proposed architectures have then addressed the problem of the trade-off between stability and transparency such as the one we presented in [35] whose architecture will be detailed in section IV-B.

\section{B. An example of a teleoperation control architecture for needle insertion}

As a pre-requisite for suturing or biopsy, needle insertion is of a great importance during surgical procedure especially in endoluminal surgery. As reported by the NOSCAR, ideally, it should be remote controlled with a good force feedback. To achieve this requirement, we proposed in [35] a teleoperation control architecture. This scheme ensures stability and provides high fidelity perceptual feedback of the interaction with the environment delivered transparently to the operator w.r.t. heterogeneous environment in the sense that the surgeon should have a good feeling of the tool/tissue interactions inside the body. The architecture, represented in figure 2, is based on a position-position structure.

As presented in figure 3, it combines the use of an active observer (AOB) with online interaction model parameter estimation. Through a haptic device, the surgeon teleoperates the instrument, fixed on a remote robot that may evolve in free or constrained space. In free space, the surgeon feels the motion of the robot due to the position/position control scheme . When contacts with tissues occur, an online environment stiffness estimation is performed using an Extended Kalman Filter. The stiffness estimation of the environment ensures the global stability of the position/position control scheme w.r.t. environment property changes. Moreover, stability and transparency frequency analysis help to improve telepresence thanks to adaptive tuning of contact stiffness.

The use of AOB combined with online interaction model parameter estimation has provided a teleoperation architecture with a good behavior for macro-scale tasks in a surgical-like environment guaranteeing the trade-off between transparency and stability. Figure 4 presents the different phases of the needle insertion and Figure 5 illustrates the corresponding experimental results. This scheme will be now adapted for endoluminal robotized surgery. 


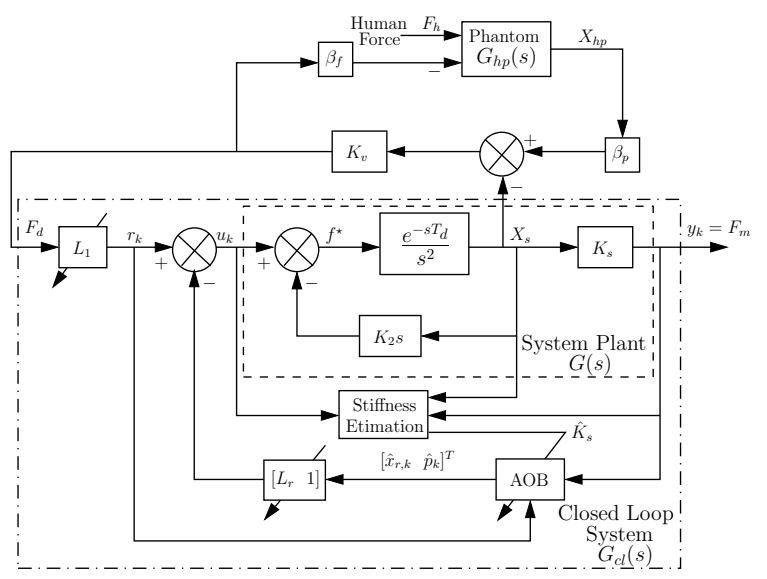

Fig. 3. Teleoperation control scheme for each Cartesian dimension. The master station (human and phantom), generates the 3D Cartesian force $F_{d}$ through the virtual coupling $K_{v} . \beta_{p}$ scales the phantom position $X_{h p}$, and $\beta_{f}$ scales back $F_{d}$ to the master station. $G(s)$ has a damping term $K_{2}$, and is controlled by AOB estimates $\left[\begin{array}{ll}\hat{x}_{r, k} & \hat{p}_{k}\end{array}\right]^{T}$ through the state feedback gain $\left[\begin{array}{ll}L_{r} & 1\end{array}\right] . K_{s}$ is the system stiffness and $L_{1}$ is the first element of $L_{r}$. $G_{c l}$ is the force closed loop. The environment stiffness is estimated $\left(\hat{K}_{s}\right)$ and used to adapt the force controller.

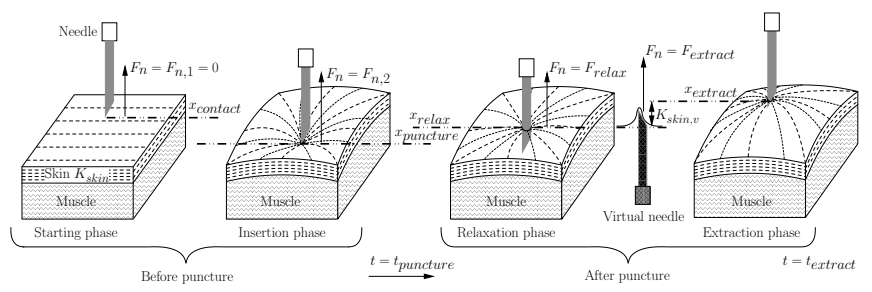

Fig. 4. Needle insertion phases into two layers soft tissue.

\section{CONCLUSION}

In this paper some control-related issues for endoluminal robotized surgery have been discussed. Mainly, two aspects have been emphasized from the technological point of view to the theoretical/practical aspects. For each of them, open questions have been highlighted. Future works will address different control issues that have been mentioned such as:

- An indepth study of performances, capabilities and limitations of the existing active-observer based teleoperation architecture,

- Increasing performances as well as robustness of this bimanual teleoperation architecture through the introduction of the predictive approach in order to take into account the different saturations that may exist (on the inputs, outputs, actuators, ...)

- Proposition of an extended architecture that may be able to achieve cooperative teleoperation, where the surgeon will be able to teleoperate multiple slave robots with only limited number of haptic interface.

\section{REFERENCES}

[1] Namjun Cho, Jerald Yoo, Seong-Jun Song, Jeabin Lee, Seonghyun Jeon, and Hoi-Jun Yoo. The human body characteristics as a signal transmission medium for intrabody communication. IEEE Transactions on Microwave Theory and Techniques, 55(5):1080-1086, 2007.

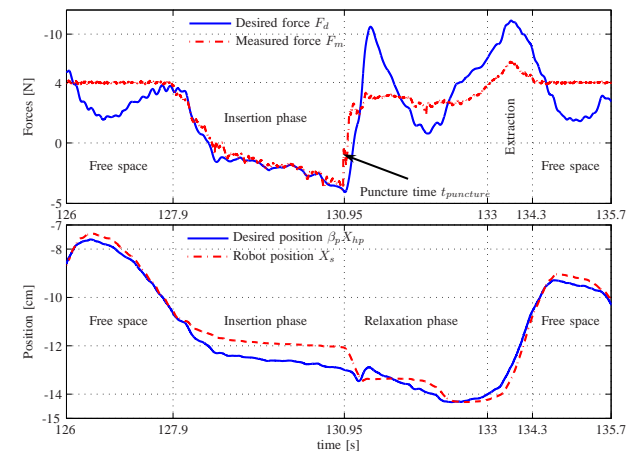

(a)

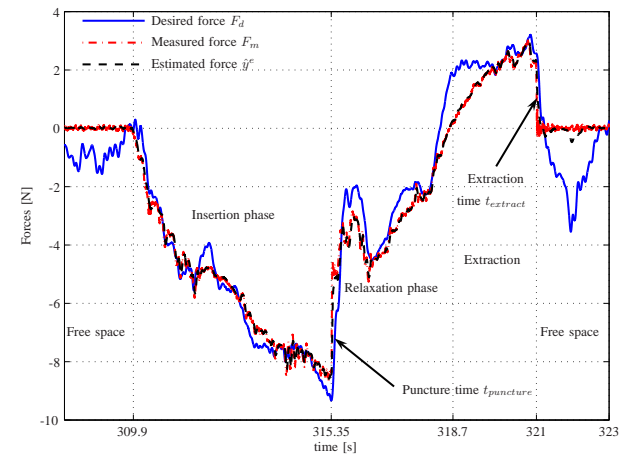

(b)

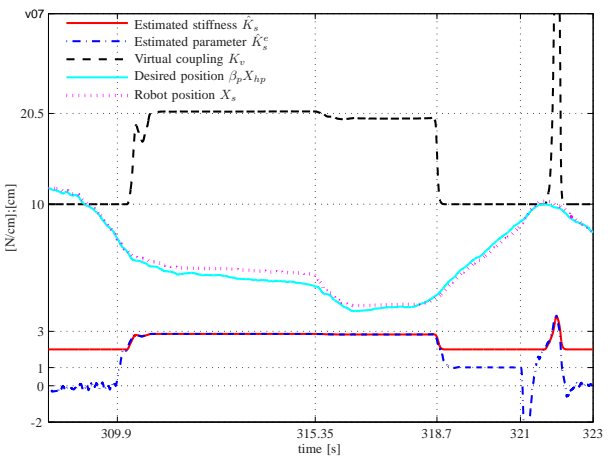

(c)

Fig. 5. Teleoperation data using the AOB controller in the field of needle insertion. (a) Without stiffness adaptation and telepresence improvement. Erroneous behavior felt at the puncture time. (b) and (c) With stiffness adaptation and telepresence improvement. (b) Force data. (b) Estimated stiffness and position data. For the clarity of the the plot, position data are shifted up.

[2] P. Dario, C. Stefanini, and A. Menciassi. Modeling and experiments on a legged microrobot locomoting in a tubular, compliant and slippery environment. In The 9th International Symposium on Experimental Robotics- ISER, 2004.

[3] A. Flynn, K. Udayakumar, D. Barret, J. McLurkin, D. Franck, and A. Shectman. Tomorrow's surgery: micromotors and microrobots for minimally invasive procedures. Journal of Minimally Invasive Therapy and Allied Technologies, 7(4):343-352, September 1998.

[4] A. Fritscher-Ravens, C.A. Mosse, and D. Mukherjee. Transgastric gastropexy and hiatal hernia repair for gerd under eus control: a porcine model. Gastrointest Endosc., 59:89-95, 2004.

[5] B. Hannaford. A design framework for teleoperators with kinesthetic feedback. IEEE Trans. Rubotics Automat., 5(4):426-434, 1989.

[6] J.P. Hespanha and A.S. Morse. Switching between stabilizing controllers. Automatica, 38(11):1905-1917, 2002.

[7] P. F. Hokayem and M. W. Spong. Bilateral teleoperation: an historical survey. Automatica, 42:2035-2057, 2006.

[8] S. B. Jagannath, S. V. Kantsevoy, and C. A. Vaughn. Peroral 
transgastric endoscopic ligation of fallopian tubes with long-term survival in a porcine model. Gastrointest Endosc., 61:449-453, 2005.

[9] Edwin W. H. Jager, Elisabeth Smela, and Olle InganŁs. Microfabricating conjugated polymer actuators. Science, 290(5496):1540-1545, November 2000.

[10] Sumiyam K., C.J. Gostout, E. Rajan, and et al. Transgastric cholecystectomy: transgastric accessibility to the gallblader improved with the semf method and a novel multibending therapeutic endoscope. Gastrointestinal endoscopy, 65:1028-1034, 2007.

[11] Aristeidis Karalis, J.D. Joannopoulos, and Marin Soljacic̀. Efficient wireless non-radiative mid-range energy transfer. Annals of Physics, 323:34-48, 2008.

[12] H. Kazerooni, T. I. Tsay, and C. L. Moore. Telefunctioning: an approach to telerobotic manipulations. In Proc. Amer. Contr. Conf, pages 2778-2783, San Diego, CA, USA, 1990.

[13] K.Y. Kim, Y. Sun, R.M. Voyles, and B.J. Nelson. Calibration of multiaxis mems force sensors using the shape from motion method. IEEE Sensors Journal, 7(3):344-351, 2007.

[14] D. A. Lawrence. Stability and transparency in bilateral teleoperation. IEEE transactions on Robotics and Automation, 9(5):624-637, 1993.

[15] A.C Lehman, K.A Berg, J. Dumpert, N.A. Wood, A.Q. Visty, M. Rentschler, S.R. Platt, S.M. Farritor, and D. Oleynikov. Surgery with cooperative robots. 13(2):95-105, March 2008.

[16] A.C Lehman, N.A. Wood, J. Dumpert, D. Oleynikov, and S.M. Farritor. Robotic natural orifice transluminal endoscopic surgery. In Proc. of the 2008 IEEE International Conference on Robotics and Automation, pages 2969-2974, May 2008.

[17] L.G. Machado and M.A. Savi. Medical application of shape memory alloys. Brazilian Journal of Medical and Biological Research, 36(6):683-691, 2003.

[18] A. Menciassi, A. Moglia, S. Gorini, G. Pernorio, C. Stefanini, and P. Dario. Shape memory alloy clamping devices of a capsule for monitoring tasks in the gastrointestinal tract. Journal of Micromechanics and Microengineering, 15(11):2045-2055, 2005.

[19] H. Ono, H. Kondo, and T. Gotoda. Endoscopic mucosal resection for treatment of early gastric cancer. Gut, 48:225-229, 2001.

[20] P. O. Park, M. Bergstrom, K. Ikeda, A. Fritscher-Ravens, and P. Swain. Experimental studies of transgastric gallbladder surgery: cholecystectomy and cholecystogastric anastomosis (videos). Gastrointest Endosc., 61:601-606, 2005.

[21] M. Pankaj Pasricha, R. A. Kozarek, and P. Swain. A next generation therapeutic endoscope: development of a novel endoluminal surgery system with "birds-eye" visualization and triangulating instruments. Gastroenterology, In press, 2009.

[22] J. Peirs, J. Clijnen, P. Herijgers, D. Reynaerts, H. Van Brussel, P. Herijgers, B. Corteville, and S. Boone. A micro optical force sensor for force feedback during minimally invasive robotic surgery. In Proc. of the 17th European Conference on Solid-State Transducers, volume 115, pages 447-455, September 2004.

[23] M. Rentschler, J. Dumpert, S.R. Platt, S. Farritor, and D. Oleynikov. Mobile in vivo robots provide sole visual feedback for abdominal exploration and cholecystectomy. Surgical Endoscopy, 20:135-138, 2006.

[24] M. Rentschler, J. Dumpert, S.R. Platt, S. Farritor, and D. Oleynikov. Natural orifice surgery with an endoluminal mobile robot. Surgical Endoscopy, 21:1212-1215, 2007.

[25] W.O. Richards, H. L. Houston, A. Torquati, L. Khaitan, M.D. Holzman, and K.W. Sharp. Paradigm shift in the management of gastroesophageal reflux disease. Ann Surg., 237:638-647, 2003.

[26] S. Seewald, S. Groth, and S. Omar. Aggressive endoscopic therapy for pancreatic necrosis and pancreatic abscess: a new safe and effective treatment algorithm (videos). Gastrointest Endosc., 62:92-100, 2005.

[27] Mohsen Shahinpoor. Potential applications of electroactive polymer sensors and actuators in mems technologies. In Proc. of the SPIE : Smart Materials, volume 4234, pages 203-214, April 2001.

[28] V. K. Sharma, M. Moirano, G. DePetris, and D.E. Fleischer. Successful ablation of barrett's esophagus with low-grade dysplasia (lgd) using barrx balloon device. In Presented at: Digestive Disease Week, pages 1881-1886, Chicago, IL, USA, 2005.

[29] Yantao Shen, Ning Xi, C.A. Pomeroy, U.C. Wejinya, and W.J. Li. An active micro-force sensing system with piezoelectric servomechanism. In Proc. of the IEEE/RSJ International Conference on Intelligent Robots and Systems, pages 2381- 2386, August 2005.

[30] Danail Stoyanov, George P. Mylonas, and Guang-Zhong Yang. Gazecontingent $3 \mathrm{~d}$ control for focused energy ablation in robotic assisted surgery. In MICCAI '08: Proceedings of the 11th International Conference on Medical Image Computing and Computer-Assisted Intervention, Part II, volume 2, pages 347-355, 2008.

[31] Michael Sultan and Richard A. Norton. Esophageal diameter and the treatment of achalasia. The American Journal of Digestive Diseases and Sciences, 14(9):611-618, 1969.

[32] Y. Sun, D.P. Potasek, D. Piyabongkarn, R. Rajamani, and B.J. Nelson. Actively servoed multi-axis microforce sensors. In Proc. of the IEEE International Conference on Robotics and Automation, pages 294-299, September 2003.

[33] F. Tendick, S. Shankar Sastry, R.S. Fearing, and M. Cohn. Applications of micromechatronics in minimally invasive surgery : Focused section on micromechatronics. IEEE/ASME transactions on mechatronics, 2(1):34-42, March 1998.

[34] Marc Simon Wegmueller, Michael Oberle, Norbert Felber, Niels Kuster, and Wolfgang Fichtner. Digital data communication through the human body for biomedical monitoring sensor. World Congress on Medical Physics and Biomedical Engineering, 14(7):608-612, 2006.

[35] W. Zarrad, P. Poignet, R. Cortesao, and O. Company. Stability and transparency analysis of a haptic feedback controller for medical applications. In Proc. IEEE Conf. on Decision and Control, pages 5767-5772, New Orleans, LA, USA, 2007.

[36] W. Zarrad, P. Poignet, R. Cortesao, and O. Company. Towards teleoperated needle insertion with haptic feedback controller. In IEEE/RSJ International Conference on Intelligent Robots and Systems, pages 1254 - 1259, November 2007. 